\title{
Is the diagnosis of systemic lupus erythematosus still a challenge? A case report
}

\author{
Alma-Mirona Nicu', Sinziana Daia-Iliescu', Cristina Aura Panea², Ruxandra Ionescư ${ }^{1}$ \\ ${ }^{1}$ Department of Internal Medicine and Rheumatology, \\ "Sfanta Maria" Clinical Hospital, Bucharest, Romania \\ ${ }^{2}$ Department of Neurology, Elias University Hospital, Romania \\ 3"Carol Davila" University of Medicine and Pharmacy, Bucharest
}

\begin{abstract}
- ABSTRACT
Systemic lupus erythematosus (SLE) is a potentially fatal autoimmune disease that remains frequently underdiagnosed due to the heterogeneity of its symptoms. Lack of specific features at the initial presentation often results in delayed diagnosis.

We present the case of a 58 years old SLE patient with renal, neurological, hematological and articular involvement and associated antiphospholipid syndrome, whose first manifestation of the disease occurred 20 years before the diagnosis could be made.

SLE is a disease with a wide array of symptoms. As such, unless a strong index of suspicion is maintained, the disease diagnosis and specific treatment may be easily delayed.
\end{abstract}

Keywords: systemic lupus erythematosus, antiphospholipid syndrome, epileptic seizures, Libman-Sacks endocarditis

\section{INTRODUCTION}

Systemic lupus erythematosus (SLE) is a disorder with a wide range of clinical and paraclinical involvement which can also have a variable course of prognosis as well as response to treatment. (1) It commonly affects women at their reproductive age. All systems and organs may not be involved initially, they are rather affected gradually, over the course of years. Diagnosis depends on characteristic clinical features and laboratory test results. Skin rash, joint involvement and oral ulcers are common features in SLE. But initial presentation of many patients is unusual because either they do not have these common features of the disease or the presentation mimics other illnesses. This can result in a delayed diagnosis or misdiagnosis (2).

\section{CASE REPORT}

We present the case of a 58 years old woman that presented in our service in July 2018 with arthralgia, vertigo, astenia and post-prandial abdominal pain. From her past medical history we focused our atten- tion on an episode of deep vein thrombosis complicated with pulmonary thromboembolism in 1998, aortic valve disease with mechanical prosthesis in 2008 , for which she is undergoing continuous treatment with acenocoumarol. She also receives antihypertensive medication. Recently, in May 2018, during a routine check-up she had significant proteinuria, microscopic hematuria, raised inflammatory markers and positive anti dsDNA and ANA. She was thus guided to our rheumatology service.

Upon admission she was afebrile, with slight skin palor, no edema, rashes or alopecia, normal heart rate and blood pressure $-120 / 70 \mathrm{mmHg}$ and $66 \mathrm{bpm}$, a grade IV/VI holosyistolic murmur at the aortic location and the lower sternal border which radiated to the axilla and the carotid artery, normal respiratory sounds, diffuse abdominal tenderness upon palpation, no neurological signs were present.

Laboratory data revealed anemia, leucopenia and thrombopenia $(\mathrm{Hb}=10.8 \mathrm{~g} / \mathrm{dl}, \mathrm{WBC}$ of $3000 / \mathrm{ul}$ and platelet count $136.000 / \mathrm{ul})$, elevated erytrocyte sedimentation rate $(E S R=109 \mathrm{~mm} / \mathrm{h})$ with normal C-reactive protein $(\mathrm{CRP}<1 \mathrm{mg} / \mathrm{L})$, low complement lev- 
els (C3=49 mg/dl), elevated levels of $\operatorname{IgG}(2299 \mathrm{mg} /$ dl), a $24 \mathrm{~h}$ urine collection showed proteinuria (1.7 $\mathrm{g} / 24 \mathrm{~h}$ ), microscopic hematuria was also present, with a slightly elevated creatinine of $1.6 \mathrm{mg} / \mathrm{dl}$. We also performed a coagulation panel that showed an prolongedaPTT of $126 \mathrm{sec}$ and the INR of 2.39. She also had a mild electrolyte imbalance, with a $\mathrm{Na}$ of $130 \mathrm{mmol} / \mathrm{l}$.

Serological studies revealed positivity for ANA (high titres), dsDNA ( $>200 \mathrm{u} / \mathrm{ml}$ ), anti cardiolipin $(>120)$ and beta-2-glycoprotein-1 (>200) antibodies and negative $\mathrm{Sm}$ antibodies. Direct Coombs test was positive. We also found a positive VDRL test with negative TPHA.

The electrocardiogram showed a grade I atrioventricular block, left ventricular hyperthrophy, and negative $\mathrm{T}$ waves in DIII and aVF derivations. The transthoracic echocardiography revealed no fluid in the pericardium, normal chamber size, an ejection fraction of $55 \%$, prostetic heart valve in aortic position with a mean gradient of $27.5 \mathrm{mmHg}$, moderate mitral stenosis and regurgitation. The thoracic radiography and the abdominal echography were within normal range. We also performed a musculoskeletal echography of the hands, were we found moderate synovitis in the radiocubital carpal joints. Ophtalmologic and ENT consults were nonspecific.

In this patient, SLE classification (SLICC 2012) criteria were accomplished when clinical criteria such as proteinuria, arthritis were summed to the previously described immunological findings - positive ANA, anti dsADN and antiphospholipid antibodies, low complement titres and positive Coombs test. The level of disease activity was high, with a SELENA-SLEDAI score of 16 . The diagnosis criteria for antphosphplipid syndrome were also met the pacient had a history of deep vein thrombosis and positive antiphospholipid antibodies.

Regarding the treatment of SLE, it should be tailored according to the severity of their disease, differentiating activity from irreversible damage, the flare severity being the one that dictates the intensity of the treatment.

We decided to start intravenous puls-theapy with methylprednisolone and hydroxycloroquine at a dose of $400 \mathrm{mg}$ a day.

On the third day of admission the pacient developed tonico-clonic seisures with loss of counciousness and transient motot deficit (left hemiplaegia), so a neurological consult was performed. The electroencephalogram showed diffuse multifocal irrita- tive activity, and the head CT was normal. Treatment with levetiracetam was initiated.

In this patient with high activity disease and multiple organ involvement, we started immunosuppression therapy according to the 2012 recommendations for the management of lupus nephritis. We decided upon treatment with mycophenolate mofetil $3 \mathrm{~g} /$ day and continued methylprednisolone ( $1.5 \mathrm{~g}$ in total) followed by oral prednisone $0.5 \mathrm{mg} / \mathrm{kg} /$ day with the intention to reduce the dose in the following months. We associated hydroxycloroquine, and continued acenocoumarol treatment and levetiracetam, as suggested by the neurologist.

Upon evaluation, after one month, because of persistent proteinuria and because the mycophenolate mofetil was not well tolerated by the patient (gastrointestinal adverse reactions), our option was to start treatment with cyclophosphamide $0.5 \mathrm{~g} / \mathrm{m} 2$, with supportive care and hydration with isotonic saline solution and glucose in order to prevent CYC toxicity. This led to a significand reduction of proteinuria at 3 months $(776 \mathrm{mg} / 24 \mathrm{~h})$ and of the disease activity score (SELENA SLEDAI 8).

\section{DISCUSSIONS}

This case delayed diagnosis SLE is particularly interesting due to the fact that the antiphospholipid syndrome has not had any clinical manifestations for such a long period of time, mainly because of the acenocumarol treatment that had been prescribed for her prosthetic mechanical valve, fact which delayed the diagnosis even further. Only after developing renal manifestations suspicion was raised and an accurate diagnosis of SLE could be made.

Antiphospholipid syndrome in lupus is not infrequent. Studies suggest that aproximately $24 \%$ of lupus patients have an associated antiphospholipid syndrome. Similarly, the antiphospholipid syndrome frequently precedes the diagnosis of lupus, often by a decade or more.2,4 In fact, a recent report indicated that positivity for antinucleosome antibodies at the time of diagnosis of antiphospholipid syndrome may help predict subsequent development of lupus. In our patient, the history of deep vein thrombosis suggests that antiphospholipid syndrome may have antedated the lupus by 8 to 10 years (3). The reverse question, what percentage of cases of antiphospholipid syndrome is associated with lupus, is not clearly answered. It should be remembered that antiphospholipid syndrome was, in fact, firstly defined in the 
setting of systemic lupus erythematosus and only later as a primary entity. It is clear that lupus is by far the most frequently associated condition (3).

In addition to the usual manifestations of antiphospholipid syndrome (APS) (deep vein thrombosis, thrombocytopenia, livedo reticularis, stroke, superficial thrombophlebitis, pulmonary embolism, transient ischemic attack) other possible antiphospholipid antibody (aPL)-related clinical manifestations include cardiac valve disease, pulmonary hypertension, avascular necrosis, adrenal insufficiency due to hemorrhagic infarction, and cognitive deficits (4).

Two major types of target organ involvement can pe found in APS, namely those affecting the heart and the central nervous system (CNS). Heart valve lesions are the most common cardiac manifestations described in patients with APS (5). The introduction of cross sectional and Doppler echocardiography has revealed a high prevalence of valvar abnormalities such as thickening, stenosis, regurgitation, and vegetations in patients with primary and secondary APS. Their frequency appears to be quite high, with up to $70 \%$ of APS patients having at least one valvular abnormality on echocardiography, as was probably the case of our patient (5).

Also, neurological involvement in antiphospholipid antibody syndrome (APS) is also common, and its occurrence increases morbidity and mortality. Patients may present variable neurological involvement, such as cerebrovascular disease, cognitive dysfunction, headache, seizures, movement disorders, multiple sclerosis-like syndrome, transverse myelitis and ocular symptoms (6).

Whether the neurological symptoms in our patient can be attributed to the APS, or are strictly related to the SLE's disease activity, is a question yet to be answered. Cerebral vascular events are frequent and can lead to neurologic deficits, seizures, or merely diminished intellectual function. In lupus, even in patients without frank cerebral infarcts, antiphospholipid antibodies and neuropsychiatric manifestations are strongly linked (7). Nevertheless, considering the serological values (low titres of complement fractions, high titers of dsDNA but also high titers of aPL), we can say that the etiology is mixed.

The same deduction can be made aboud the renal involvement in this patient. Antiphospholipid syn- drome may also occur with any class of lupus nephritis (LN). There is no association between the class of lupus nephritis and antiphospholipid syndrome, neither with activity scores. There also is no correlation between antiphospholipid antibody titers and anti-DNA antibody or complement levels. Clinically evident lupus nephritis is a frequent form of organ involvement in up to $50 \%$ of patients with SLE and carries a worse prognosis than non-renal disease. The vast majority of patients who develop LN do so within 5 years of SLE diagnosis, few do so subsequently (8).

Another thing to take into consideration is the patient's hyponatremia. In the absecnce of structural brain lesions (head CT was clear) or evidence of heart or liver failure, the hyponatremia may be secondary to innapropriate secretion of antidiuretic syndrome. The SIADH related to SLE is mentioned in small studies and case reports, most of the cases being associated with neurolupus, thrombotic or vasculitic lesions (9).

\section{CONCLUSIONS}

Systemic lupus erythematosus (SLE) and antiphospholipid syndrome (APS) or Hughes syndrome are probably the most important paradigm of systemic autoimmune disease. SLE is known as the great imitator, because its symptoms mimic many other illnesses (10). Early diagnosis is important, but the lack of a gold standard test to confirm diagnosis results in delays or misdiagnosis.

APS is increasingly recognized as a multisystem disease, the clinical expression of which may include cardiac, neurological, hematological, cutaneous and other manifestations. There is transition from APS to SLE with secondary APS. Special attention should be given to secondary APS patients when they are submitted to high-risk events: from $7-10 \%$ patients with PAPS may go on to develop SLE (10). Despite updates of the diagnostic criteria of both diseases, the diagnosis of SLE and APS remains difficult to make.

In conclusion, despite advances in the understanding of the immunopathogenic and clinical aspects of SLE, early diagnosis remains a diagnostic and therapeutic challenge.

Conflict of interest: none declared Financial support: none declared 


\section{REFERENCES}

1. Montero-Olvera PR, Berebichez-Fridman R, Velázquez-Álvarez L, Ríos-Morales JR, Rodríguez-Guiza MA. Late diagnosis of systemic lupus erythematosus and antiphospholipid syndrome in an older woman with psychosis: a case report and review of the literature. Clin Case Rep. 2017;5(11):1819-1825. Published 2017 Sep 26. doi:10.1002/ccr3.1185

2. Pramanik B1. Diagnosis of systemic lupus erythematosus in an unusual presentation: what a primary care physician should know, Curr Rheumatol Rev. 2014;10(2):81-6

3. Antiphospholipid Syndrome in Systemic Lupus Erythematosus, Gary S. Hill and Dominique Nochy, JASN Sept 2007, 18 (9) 24612464; DOI: https://doi.org/10.1681/ASN.2007030257

4. Sciascia S, Baldovino S, Schreiber K, et al. Thrombotic risk assessment in antiphospholipid syndrome: the role of new antibody specificities and thrombin generation assay. Clin Mol Allergy. 2016;14:6. Published 2016 Jul 15. doi:10.1186/s12948-016-0043-2

5. Krause I, Lev S, Fraser A, et al, Close association between valvar heart disease and central nervous system manifestations in the antiphospholipid syndrome, Annals of the Rheumatic Diseases 2005;64:1490-1493

6. Farzaneh-Far A, Roman MJ, Lockshin MD, Devereux RB, Paget SA, Crow MK, Davis A, Sammaritano L, Levine DM, Salmon JE:
Relationship of antiphospholipid antibodies to cardiovascular manifestations of systemic lupus erythematosus. Arthritis Rheum 54 : 3918 -3925, 2006

7. Sanna G, Bertolaccini ML, Cuadrado MJ, Laing H, Khamashta MA, Mathieu A, Hughes GR: Neuropsychiatric manifestations in systemic lupus erythematosus: Prevalence and association with antiphospholipid antibodies. J Rheumatol 30 : 985 -992, 2003

8. Alexandre AR, Carreira PL, Isenberg DA, Very delayed lupus nephritis: a report of three cases and literature review, Lupus Science \& Medicine 2018;5:e00241. doi: 10.1136/ lupus-2017-000241

9. Ioana Saulescu, Sanziana Daia-lliescu, C.L. Constantinescu, Andreea Borangiu, Casandra Buzatu, Ruxandra Ionescu, Daniela Opris-Belinski, Severe hyponatremia, hypertension, acute deterioration of renal function in a young female with lupus nephritis RJR vol XXVI 2017

10. Ljudmila Stojanovich, Diagnostic challenges in systemic lupus erythematosus and antiphospholipid syndrome, DOI: 10.21767/2572-5548-C1-002 